\title{
ANÁLISE DE AGRUPAMENTO DO IMC E DO IAC E DA RELAÇÃO IAC/IMC EM INDIVÍDUOS COM IDADE ENTRE 6 A 15 ANOS
}

\author{
Julião Soares de Souza Lima Junior ${ }^{1}$ \\ Larissa Costa de Souza Lima² \\ Thais Gomes Oliveira Laia ${ }^{3}$ \\ Alessandra Fagioli da Silva ${ }^{4}$
}

Resumo: Conhecer as relações entre dados antropométricos e o estado nutricional das pessoas ajuda no entendimento das tendências em uma população. Este trabalho teve o objetivo de determinar em 62 jovens com idade entre 6 a 15 anos o IMC, o IAC e a relação entre IAC/IMC e utilizar método para agrupamento dos indivíduos. Os dados foram analisados por estatística descritiva e o método de agrupamento Joining. O IMC, o IAC e a Relação IAC/IMC, apresentaram os valores médios de 18,88; 27,62 e 1,51, respectivamente. Os grupos 1 e 2 apresentaram médias maiores que a média geral dos dados para o IMC. Os grupos 2, 3 e 4 apresentaram maiores valores médios que a média geral dos dados para o IAC. Os grupos 3 e $4 \mathrm{com}$ média maior que a media geral para a relação IAC/IMC. Maiores relações de IAC/IMC ocorrem para indivíduos que apresentam menores valores de IMC e os maiores de IAC. O IMC aumenta com a idade e o IAC diminui à medida que a idade aumenta.

Palavras-chave: Saúde coletiva; Bem-estar; Alimentação saudável.

\footnotetext{
${ }^{1}$ Medicina/Centro Universitário de Caratinga-UNEC, Brasil. E-mail: juliaosoaresneuroligia@outlook.com.

${ }^{2}$ Odontologia/Universidade Vale do Rio Doce-UNIVALE, Brasil. E-mail: larissa_csI@hotmail.com.

3 Odontologia/Universidade Vale do Rio Doce-UNIVALE, Brasil. E-mail: tg-laia@bol.com.br.

${ }^{4}$ Agronomia/Universidade Estadual do Norte do Paraná-UENP, Brasil. E-mail: alefagioli@hotmail.com.
} 